\title{
Experimental and theoretical study of magnetic ordering and local atomic polarization in $\mathrm{Ru}$-substituted $\mathrm{Lu}_{2} \mathrm{Fe}_{17}$
}

\author{
E. A. Tereshina, ${ }^{1}$ O. Isnard, ${ }^{2}$ A. Smekhova, ${ }^{3,4}$ A. V. Andreev, ${ }^{1}$ A. Rogalev, ${ }^{4}$ and S. Khmelevskyi ${ }^{5}$ \\ ${ }^{1}$ Institute of Physics, Academy of Sciences of the Czech Republic, Prague 18221, Czech Republic \\ ${ }^{2}$ Institut Néel, CNRS/Université Grenoble Alpes, BP166, Grenoble 38042, France \\ ${ }^{3}$ Faculty of Physics, M. V. Lomonosov Moscow State University, Moscow 119991, Russia \\ ${ }^{4}$ European Synchrotron Radiation Facility, BP 220, Grenoble 38043, France \\ ${ }^{5}$ Vienna University of Technology, Department of Applied Physics, Vienna 1040, Austria
}

(Received 14 November 2013; revised manuscript received 18 February 2014; published 21 March 2014)

\begin{abstract}
The distribution of magnetic moments in the iron-rich intermetallic compound $\mathrm{Lu}_{2} \mathrm{Fe}_{17}$ with $\mathrm{Fe}$ partly substituted by $\mathrm{Ru}$ has been investigated on $\mathrm{Lu}_{2} \mathrm{Fe}_{16.5} \mathrm{Ru}_{0.5}$ by using several techniques. The crystal structure and temperature evolution of the magnetic structure was studied using high resolution and high flux neutron powder diffractometers. In contrast to the parent $\mathrm{Lu}_{2} \mathrm{Fe}_{17}$, which transforms on cooling from an antiferromagnetic to a ferromagnetic state, $\mathrm{Lu}_{2} \mathrm{Fe}_{16.5} \mathrm{Ru}_{0.5}$ is an antiferromagnet (Néel temperature $T_{\mathrm{N}}=208 \mathrm{~K}$ ) down to the lowest temperatures. The magnetic polarization at the lutetium $L_{2,3}$ absorption edges, iron $K$ and ruthenium $L_{2}$ edges was studied by $\mathrm{x}$-ray magnetic circular dichroism (XMCD) on a single crystal of $\mathrm{Lu}_{2} \mathrm{Fe}_{16.5} \mathrm{Ru}_{0.5}$ in a magnetic field of $3 \mathrm{~T}$, i.e., above the field-induced metamagnetic transition. The Ru XMCD signal proves the existence of induced magnetic polarization parallel to the dominant Fe sublattice magnetization. A corresponding theoretical analysis in the framework of the local spin density formalism is provided in order to discern the structural disorder effects and magnetism in the system.
\end{abstract}

DOI: 10.1103/PhysRevB.89.094420

PACS number(s): 75.25.-j, 78.70.Dm, 71.20.-b

\section{INTRODUCTION}

It has long been known that the magnetic moments of transition metals are intimately tied to the local density of electronic states and vary strongly with the local coordination and type of magnetically active neighboring atoms. This is especially pronounced in the $4 d$ elements, which can develop a magnetic moment when dissolved substitutionally in the strong and weak $3 d$ ferromagnets by way of polarizing the $d$ electrons [1-5]. There exists a large amount of information on the electronic and magnetic properties of diluted $\mathrm{Fe}$ pseudobinary alloys. For instance, $\mathrm{Ru}$ impurity in iron host has been shown to carry a noticeable magnetic moment $[1,5]$. However, experimental and computational data on Ru placed in a complex Fe matrix of binary and ternary alloys are scarce except, perhaps, recent major interest in modifying the properties of superconducting iron-based compounds by way of substituting $\mathrm{Ru}$ for $\mathrm{Fe}[6,7]$. In this case, the substitution was shown to be isoelectronic and acting in a similar fashion to magnetic dilution [7].

The rare earth $R$-Fe intermetallics-materials with hard magnetic and magnetostrictive properties [8,9]—are suitable objects for studying the $4 d$ dilution on complex $3 d$-electron sublattice magnetism as the presence of $R$ is driving the systems to the structures that are more closely packed, thus exhibiting features with similarities to fcc iron [10]. Strong magnetovolume effects in the most iron rich of all $R$-Fe binaries, $R_{2} \mathrm{Fe}_{17}$, are reflected in (i) remarkably low ordering temperatures ranging from as little as $240 \mathrm{~K}$ in the compound with $R=$ Ce to $480 \mathrm{~K}$ for that with $\mathrm{Gd}$; (ii) thermal expansion anomalies in the vicinity of Curie point $\left(T_{\mathrm{C}}\right)[11,12]$; and (iii) large pressure dependence of $T_{\mathrm{C}}$ and noncollinear

\footnotetext{
*Corresponding author: teresh@fzu.cz
}

magnetic structures observed in some of the compounds under ambient $[13,14]$ and hydrostatic $[15,16]$ pressure. A compound with the smallest unit cell volume among $R_{2} \mathrm{Fe}_{17}$ and a completely filled $4 f$ shell of the rare earth $\mathrm{Lu}_{2} \mathrm{Fe}_{17}$ is ideal for all intents of gaining the basic understanding of the $3 d$-electron sublattice nature in these Fe-rich rare earth intermetallics.

The crystal structure of $\mathrm{Lu}_{2} \mathrm{Fe}_{17}$ (hereafter, we use this widely accepted notation for this modified-by-doping compound) is partially disordered and departs from the ideal $\mathrm{Th}_{2} \mathrm{Ni}_{17}$ by having an excess of Fe toward the ratio 2:19 (see Fig. 1 and Ref. [17]). The magnetic structure, while ferromagnetic $(\mathrm{F})$ at low temperatures, is helimagnetic above $\Theta_{\mathrm{T}}$ $\sim 107-142 \mathrm{~K}$ (depending on the cooling-heating conditions), and the magnetic helix holds up to the Néel temperature $T_{\mathrm{N}} \sim$ $270 \mathrm{~K}[11,14]$.

Lattice distortions may significantly alter the magnetic properties of $\mathrm{Lu}_{2} \mathrm{Fe}_{17}$ and affect the magnetic ordering temperatures. For example, a pristine sample can be pushed into the antiferromagnetic (AF) state down to lowest temperatures when hydrostatically or uniaxially compressed with the applied pressure above $0.3 \mathrm{GPa}$ [18]. Exposure of $\mathrm{Lu}_{2} \mathrm{Fe}_{17}$ to chemical pressure (expansion or contraction of the lattice) caused by inserting suitable interstitial and substituting atoms was investigated in Refs. [19-24]. Minor expansion of the unit cell $(\sim 0.4 \%)$ by absorbing light interstitial elements, such as hydrogen, was shown to fully reinforce the ferromagnetic state in $\mathrm{Lu}_{2} \mathrm{Fe}_{17} \mathrm{H}$ [20]. The robustness of the ferromagnetic phase against various magnetic and nonmagnetic substitutions at the Fe sites has been reported for $\mathrm{Lu}_{2} \mathrm{Fe}_{17-x} M_{x}(M=\mathrm{Cr}, \mathrm{Mn}$, $\mathrm{Ni}, \mathrm{Si}$, or $\mathrm{Al}$ ) solid solutions [21-23]. In fact, strengthening of the ferromagnetic interactions manifested by dramatically increased ordering temperatures was observed in all modifiedby-doping compounds even for the case of the decreased unit cell volume while the AF phase ceased to exist. This suggested that the effect is not merely due to a change in volume 


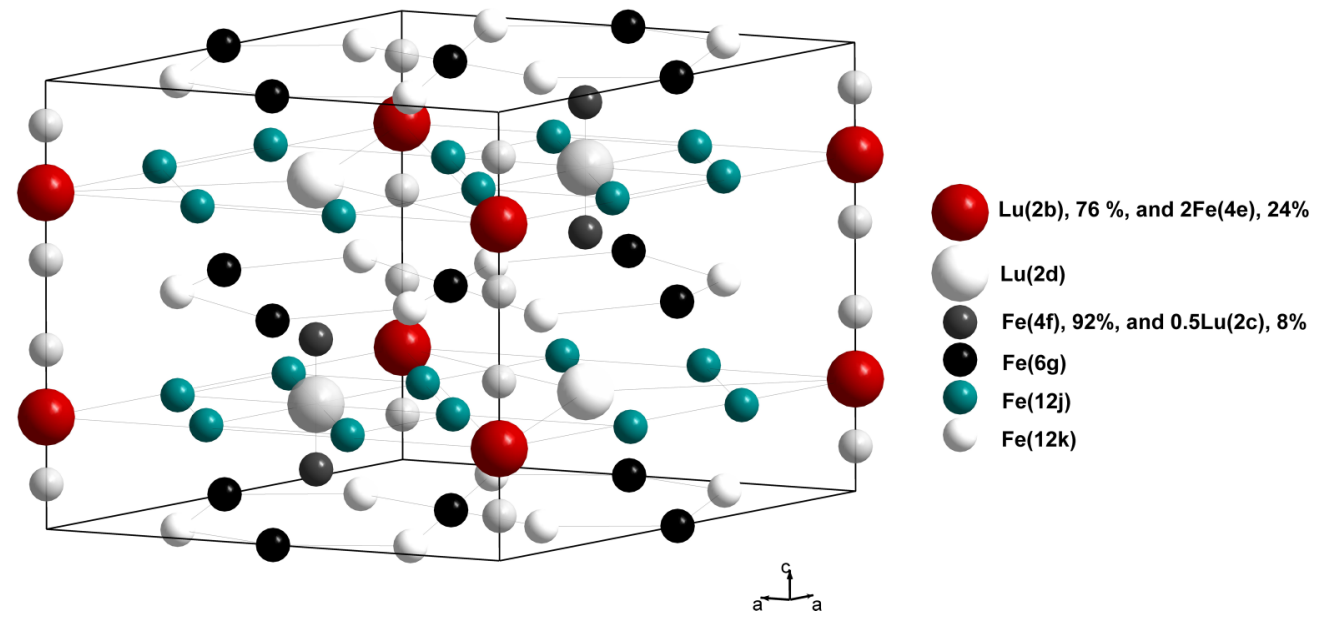

FIG. 1. (Color onlinie) The semi-ideal hexagonal $\mathrm{Th}_{2} \mathrm{Ni}_{17}$ crystal structure of $\mathrm{Lu}_{2} \mathrm{Fe}_{17}$. Shared atomic positions in a party disordered structure are shown schematically: $\mathrm{Lu}(2 c)$ is placed in between two $\mathrm{Fe}(4 f)$ atoms, to which it is substituted in a random fashion; $\mathrm{Fe}(4 e)$ pairs substitute $\operatorname{Lu}(2 b)$ randomly in the chains located along the $c$ axis.

but is also due to decreased local coordination number of Fe ions.

With this in mind, the AF state stabilized at all temperatures when $\mathrm{Fe}$ atoms in $\mathrm{Lu}_{2} \mathrm{Fe}_{17}$ are partly substituted by a $4 d$ metal $\mathrm{Ru}[25,26]$ is striking. For the alloy from the homogeneity range border $\mathrm{Lu}_{2} \mathrm{Fe}_{16} \mathrm{Ru}, T_{\mathrm{N}}$ decreased more than twofold despite a $\sim 0.7 \%$ volume boost [26]. Assuming preserved carrier concentration, one expects the $\mathrm{Ru}$ substitution for $\mathrm{Fe}$ to affect the bandwidth and hybridization in $\mathrm{Lu}_{2} \mathrm{Fe}_{17-x} \mathrm{Ru}_{x}$ in a similar way as application of "negative" pressure. However, larger $\mathrm{Ru}$ atoms, as compared to $\mathrm{Fe}$, prefer certain lattice sites in the $\mathrm{Th}_{2} \mathrm{Ni}_{17}$ type of crystal structure [25], thus affecting locally the atoms positions. Unambiguously defined structural data appears highly desirable for the study of the idiosyncrasies of magnetism in this system.

For 1.5 at.\% of $\mathrm{Ru}$ impurity in iron host, the neutrondiffraction study and the electronic structure calculations showed that the magnetic moment of $\mathrm{Ru}$ is $(0.9 \pm 0.5)$ $\mu_{B}[1,5]$. At $\mathrm{Ru}$ concentrations of an order higher, the ${ }^{57} \mathrm{Fe}$ Mössbauer absorption spectroscopy experiment suggested the AF ordering of the Ru-Fe solutions [27]. In order to obtain information on the local distribution of host and impurity magnetic moments in the complex $\mathrm{Lu}_{2} \mathrm{Fe}_{17-x} \mathrm{Ru}_{x}$ alloys, a $\mathrm{Lu}_{2} \mathrm{Fe}_{16.5} \mathrm{Ru}_{0.5}$ compound with $T_{\mathrm{N}}$ of $208 \mathrm{~K}$ [26] was chosen for the detailed study. (It is experimentally desirable that the defect concentration is high enough to allow an accurate measurement. On the other hand, $\mathrm{Lu}_{2} \mathrm{Fe}_{16.5} \mathrm{Ru}_{0.5}$ featuring a field-induced first-order metamagnetic transition in the magnetic field below 1 Tesla suited the measurement conditions.) Powerful means for studying the $\mathrm{Lu}_{2} \mathrm{Fe}_{16.5} \mathrm{Ru}_{0.5}$ compound were provided by combination of powder-neutron diffraction for the crystal and magnetic structure determination with the spectroscopic spin-dependent methods such as X-ray magnetic circular dichroism (XMCD) and x-ray-absorption near-edge structure (XANES) obtained on a single-crystalline specimen. This allowed us to probe induced local magnetic polarizations at nominally nonmagnetic lutetium and ruthenium and their coupling to the iron sublattice magnetization, thus unraveling the contribution of each element into the overall AF behavior of $\mathrm{Lu}_{2} \mathrm{Fe}_{16.5} \mathrm{Ru}_{0.5}$. To isolate the roles of structural disorder and magnetism in this important intermetallic system, the site dependencies of magnetic moments and exchange interactions in $\mathrm{Lu}_{2} \mathrm{Fe}_{16.5} \mathrm{Ru}_{0.5}$ are obtained theoretically in the framework of the local spin density formalism for the ideal $\mathrm{Th}_{2} \mathrm{Ni}_{17}$ structure model.

\section{EXPERIMENTAL DETAILS}

The magnetic and spectroscopic studies were performed on a single-crystalline specimen of $\mathrm{Lu}_{2} \mathrm{Fe}_{16.5} \mathrm{Ru}_{0.5}$ grown by a modified Czochralski method in a tri-arc furnace (the details can be found in Ref. [26]). The back Laue patterns were used to check the monocrystalline state and to orient the crystal for cutting the samples. In order to exclude possible composition difference within the crystal length, powder samples for the neutron diffraction were prepared from the top and bottom parts of the crystal and from polycrystalline ingots. The reference magnetization isotherm along the easy $a$ axis of a $\mathrm{Lu}_{2} \mathrm{Fe}_{16.5} \mathrm{Ru}_{0.5}$ single crystal was obtained at $9 \mathrm{~K}$ on a SQUID magnetometer (Quantum Design, USA).

The crystal structure of $\mathrm{Lu}_{2} \mathrm{Fe}_{16.5} \mathrm{Ru}_{0.5}$ was examined at $300 \mathrm{~K}$ using the high resolution neutron-powder diffractometer D1A operating with a $122^{\circ}$ take-off angle of monochromator at the Institut Laue-Langevin (Grenoble, France). The measurements were carried out at a wavelength $\lambda=1.91 \AA$, selected by the (115) reflection of a germanium monochromator. During the experiment, a cylindrical vanadium sample holder with a 7-mm inner diameter was used. The neutron detection was performed with a set of $6^{\circ}$ spaced ${ }^{3} \mathrm{He}$ counting tubes. Complete diffraction patterns were obtained by scanning the entire $2 \theta$ range. To study the magnetic structure of $\mathrm{Lu}_{2} \mathrm{Fe}_{16.5} \mathrm{Ru}_{0.5}$, the temperature-dependent measurements at 2-300 $\mathrm{K}$ were performed on a double-axis high intensity powder diffractometer D1B $(\lambda=2.52 \AA)$ with the use of a standard Orange cryostat. Diffraction patterns were refined by the Rietveld analysis with the FullProf/WinPLOTR software using the coherent scattering lengths of $0.945 \cdot 10^{-12} \mathrm{~cm}$, 
$0.703 \cdot 10^{-12} \mathrm{~cm}$, and $0.7210 \cdot 10^{-12} \mathrm{~cm}$ for $\mathrm{Fe}, \mathrm{Ru}$, and $\mathrm{Lu}$ nucleus, respectively.

The XANES and XMCD measurements on a singlecrystalline plate-shaped $\mathrm{Lu}_{2} \mathrm{Fe}_{16.5} \mathrm{Ru}_{0.5}$ sample with approximately $5 \times 7 \times 2 \mathrm{~mm}^{3}$ dimensions were carried out at the beamline ID12 of the European Synchrotron Radiation Facility (ESRF) in Grenoble, France. The magnetic field of $\pm 3 \mathrm{~T}$ applied along the easy $a$ axis of the crystal was large enough to induce appreciable bulk magnetization (i.e., to surpass the metamagnetic transition) and to overcome shape anisotropy. The XMCD spectra were derived as a direct difference of XANES spectra recorded with left and right circularly polarized $\mathrm{x}$ rays when the incident beam was collinear with an external magnetic field. The spectra were obtained at low temperatures $9 \pm 1 \mathrm{~K}$ (denoted as LT throughout the absorption data plots) in the back-scattering geometry with total fluorescence yield detection mode. To ensure that the result was free of any experimental artifacts, the spectra were recorded for two opposite magnetic field directions. The edge heights at the XANES spectra were normalized to unity at the Fe $K$ and $\mathrm{Lu} L_{3}$ absorption edges and to 0.5 at the $\mathrm{Lu}(\mathrm{Ru})$ $L_{2}$ edge. The XMCD data at the $\mathrm{Lu} L_{2,3}$ and $\mathrm{Ru} L_{2}$ edge were then additionally corrected for the incomplete circular polarization rate of incoming $\mathrm{x}$ rays (for the Fe $K$ absorption, the discrepancy was within $\sim 5 \%$ as compared to raw XMCD data). All recorded spectra were corrected for self-absorption except those for $\mathrm{Ru} L_{2}$, where the corrections were found to be negligible. To do so, the chemical composition, density, practically infinite thickness of the single-crystalline bulky sample, background contributions, the angle of incidence of the X-ray beam, and the solid angle of the detector were taken into account [28]. The XMCD field dependency presented below was measured following the maximum of the XMCD Fe $K$-edge signal.

\section{CRYSTAL STRUCTURE}

The room-temperature powder-diffraction pattern of $\mathrm{Lu}_{2} \mathrm{Fe}_{16.5} \mathrm{Ru}_{0.5}$ is shown in Fig. 2. The observed Bragg peaks can be indexed with the hexagonal $P 6_{3} / m m c$ space group $\left(\mathrm{Th}_{2} \mathrm{Ni}_{17}\right.$ type of crystal structure) and with the lattice parameters $a=8.406 \AA$ and $c=8.301 \AA$. Traces of an impurity $\alpha$-Fe phase (no more than 1 wt. \%) have been detected. Since the experiment was performed at 300 K, i.e., well above the magnetic ordering temperature, the Rietveld refinement procedure allowed us to determine all atomic positions in the crystal lattice of $\mathrm{Lu}_{2} \mathrm{Fe}_{16.5} \mathrm{Ru}_{0.5}$. An ideal $\mathrm{Th}_{2} \mathrm{Ni}_{17}$ structure model with full occupation of the sites has led to an unsatisfactory result. A final value of fit quality $\chi^{2}=9.2$ was obtained for up to $24 \%$ of additional $\mathrm{Fe}-\mathrm{Fe}$ pairs (4e sites) substituted for Lu (2b) located along the $c$ axis (shown schematically in Fig. 1). Reverse procedure of adding $\mathrm{Lu}(2 c)$ atoms instead of $4 f \mathrm{Fe}$ dumbbells, as suggested by Givord et al. [17], has also provided nonzero occupancy. The full list of structural parameters is reported in Table I. The refined sample composition is $\mathrm{Lu}_{1.84} \mathrm{Fe}_{16.84} \mathrm{Ru}_{0.48}$.

Further, the addition of $\mathrm{Fe} 4 e$ dumbbells around the $2 b$ $\mathrm{Lu}$ atoms was found to induce distortions nearby the $12 j$ $\mathrm{Fe}$ site. The latter has therefore split into two sites with 68 and $32 \%$ occupancies, providing two configurations of the

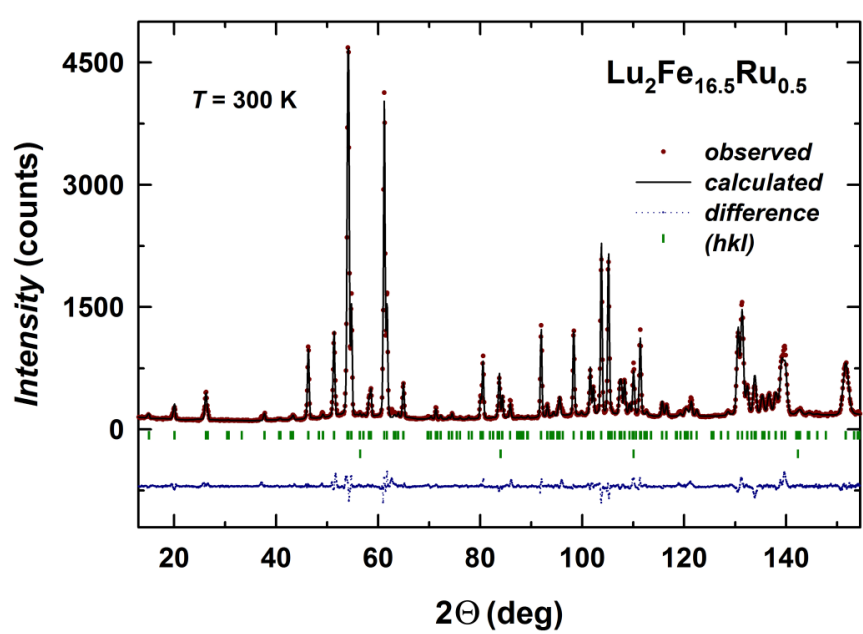

FIG. 2. (Color online) Powder neutron-diffraction pattern ( $\lambda=1.91 \AA$ ) from $\mathrm{Lu}_{2} \mathrm{Fe}_{16.5} \mathrm{Ru}_{0.5}$ at room temperature. Observed (calculated) profiles are given by the dots (solid curve), and the calculated positions are marked, including the minor $\alpha$-Fe contaminant. The difference between the observed and calculated patterns is shown at the bottom of the figure. The agreement factors of the fit are $\chi^{2}=9.2\left(R_{\exp }=3.9\right), R_{\mathrm{Bragg}}=5.7$, and $R_{\mathrm{F}}=4.9(\%)$.

$(x, y)$ plane. Furthermore, the decomposed $12 j$ crystallographic position involved sequential splitting of the $12 k$ Fe site. In the course of the diffraction pattern analysis, the occupation factors for Ru were allowed to vary at different sites. Due to a significant difference in the scattering lengths of $\mathrm{Fe}$ and $\mathrm{Ru}$ nuclei, preferential Ru occupation for the $12 k_{(2)}$ Wyckoff position in the crystal structure was clearly observed (Table I). The $\mathrm{Ru}$ role at the $12 k_{(2)}$ sites is rather intricate. Despite an $\sim 0.1 \%$ increase of the $c$-axis lattice constant and overall unit cell expansion upon the $\mathrm{Ru}$ doping in $\mathrm{Lu}_{2} \mathrm{Fe}_{17}$, the shortest

TABLE I. Structural parameters of $\mathrm{Lu}_{2} \mathrm{Fe}_{16.5} \mathrm{Ru}_{0.5}$ at room temperature from the neutron powder-diffraction experiment. Estimated standard deviations are given in parentheses.

\begin{tabular}{|c|c|c|c|c|c|}
\hline \multirow[b]{2}{*}{ Atom } & \multirow[b]{2}{*}{ Site } & \multicolumn{3}{|c|}{ Coordinates } & \multirow[b]{2}{*}{ Occ. $(\%)$} \\
\hline & & $x$ & $y$ & $z$ & \\
\hline $\mathrm{Lu}_{1}$ & $2 b$ & 0 & 0 & $1 / 4$ & $76(1)$ \\
\hline $\mathrm{Lu}_{2}$ & $2 d$ & $1 / 3$ & $2 / 3$ & $3 / 4$ & 100 \\
\hline $\mathrm{Lu}_{3}$ & $2 c$ & $1 / 3$ & $2 / 3$ & $1 / 4$ & $8(1)$ \\
\hline $\mathrm{Fe}_{1}$ & $4 f$ & $1 / 3$ & $2 / 3$ & $0.1084(5)$ & $92(1)$ \\
\hline $\mathrm{Ru}_{1}$ & $4 f$ & $1 / 3$ & $2 / 3$ & $0.1084(5)$ & 0 \\
\hline $\mathrm{Fe}_{2}$ & $6 g$ & $1 / 2$ & 0 & 0 & 100 \\
\hline $\mathrm{Ru}_{2}$ & $6 g$ & $1 / 2$ & 0 & 0 & 0 \\
\hline $\mathrm{Fe}_{3(1)}$ & $12 j_{(1)}$ & $0.3355(9)$ & $-0.0430(8)$ & $1 / 4$ & $68(1)$ \\
\hline $\mathrm{Ru}_{3(1)}$ & $12 j_{(1)}$ & $0.3355(9)$ & $-0.0430(8)$ & $1 / 4$ & 0 \\
\hline $\mathrm{Fe}_{3(2)}$ & $12 j_{(2)}$ & $0.3027(14)$ & $-0.0191(14)$ & $1 / 4$ & $32(1)$ \\
\hline $\mathrm{Ru}_{3(2)}$ & $12 j_{(2)}$ & 0.3027 (14) & $-0.0191(14)$ & $1 / 4$ & 0 \\
\hline $\mathrm{Fe}_{4(1)}$ & $12 k_{(1)}$ & $0.1658(5)$ & $2 x$ & $-0.0228(2)$ & $69(1)$ \\
\hline $\mathrm{Ru}_{4(1)}$ & $12 k_{(1)}$ & $0.1658(5)$ & $2 x$ & $-0.0228(2)$ & 0 \\
\hline $\mathrm{Fe}_{4(2)}$ & $12 k_{(2)}$ & $0.1658(5)$ & $2 x$ & $0.0007(2)$ & $23(1)$ \\
\hline $\mathrm{Ru}_{4(2)}$ & $12 k_{(2)}$ & $0.1658(5)$ & $2 x$ & $0.0007(2)$ & $8(1)$ \\
\hline $\mathrm{Fe}_{5}$ & $4 e$ & 0 & 0 & $0.1001(24)$ & $24(1)$ \\
\hline $\mathrm{Ru}_{5}$ & $4 e$ & 0 & 0 & $0.1001(24)$ & 0 \\
\hline
\end{tabular}


Fe-Fe distances (Fe atoms at $4 f$ sites) along the $c$ axis have decreased from $2.39 \AA$ in $\mathrm{Lu}_{2} \mathrm{Fe}_{17}$ [17] down to $2.35 \AA$ in $\mathrm{Lu}_{2} \mathrm{Fe}_{16.5} \mathrm{Ru}_{0.5}$. This is owed to the larger ionic radius of $\mathrm{Ru}$ and a farther displacement of the $\mathrm{Fe} 12 k_{(2)}$ atoms from their initial position.

It is critical to note that lattice parameters in $\mathrm{Lu}_{2} \mathrm{Fe}_{16.5} \mathrm{Ru}_{0.5}$ display an anomalous temperature behavior, a feature typical of the $R_{2} \mathrm{Fe}_{17}$-type compounds [12,29]. Deduced from the powder neutron-diffraction experiment at $2 \mathrm{~K}$ (see Sec. IV.C for details) are the lattice constants of $\mathrm{Lu}_{2} \mathrm{Fe}_{16.5} \mathrm{Ru}_{0.5}$, $a=8.389 \AA$ and $c=8.315 \AA$. One can see that the $c$ parameter exhibits a negative thermal expansion when the temperature is lowered. Therefore, the lattice constants obtained at $2 \mathrm{~K}$ will be used for the electronic structure calculations.

\section{PHYSICAL PROPERTIES}

\section{A. Iron $K$-edge XANES and XMCD results}

To understand the origin of magnetic order in $\mathrm{Lu}_{2} \mathrm{Fe}_{16.5} \mathrm{Ru}_{0.5}$, we probed the electronic states of iron by recording the XANES and XMCD spectra at the $\mathrm{Fe} K$ absorption edge in the magnetic field of $\mu_{0} H= \pm 3 \mathrm{~T}$ applied along the easy $a$ axis of a $\mathrm{Lu}_{2} \mathrm{Fe}_{16.5} \mathrm{Ru}_{0.5}$ single crystal. Figure 3 shows the normalized Fe $K$-edge XANES spectrum of $\mathrm{Lu}_{2} \mathrm{Fe}_{16.5} \mathrm{Ru}_{0.5}$. This spectrum reflects predominantly the $4 p$ empty states of Fe probed via the $1 s-4 p$ dipolar transitions. The spectrum is characteristic of Fe metal and does not exhibit any feature that could be attributed to the presence of $\mathrm{Fe}$ oxides. For comparison, we have also reproduced [Fig. 3(a)] XANES spectrum of an iron foil taken from Ref. [30]. The Fe $K$-edge data of $\alpha$-Fe exhibit a characteristic kink at the onset of the absorption edge at $E \sim 7112-7116 \mathrm{eV}$ (and corresponding peaks in XMCD spectra)—a feature usually assigned to the $1 s \rightarrow 3 d$ quadrupolar transitions [31]. By comparing experimental data for $\mathrm{Lu}_{2} \mathrm{Fe}_{16.5} \mathrm{Ru}_{0.5}$ with that of $\alpha$-Fe, a well-pronounced pre-edge peak in our singlecrystalline sample situated at $E \sim 7115 \mathrm{eV}$ becomes apparent.

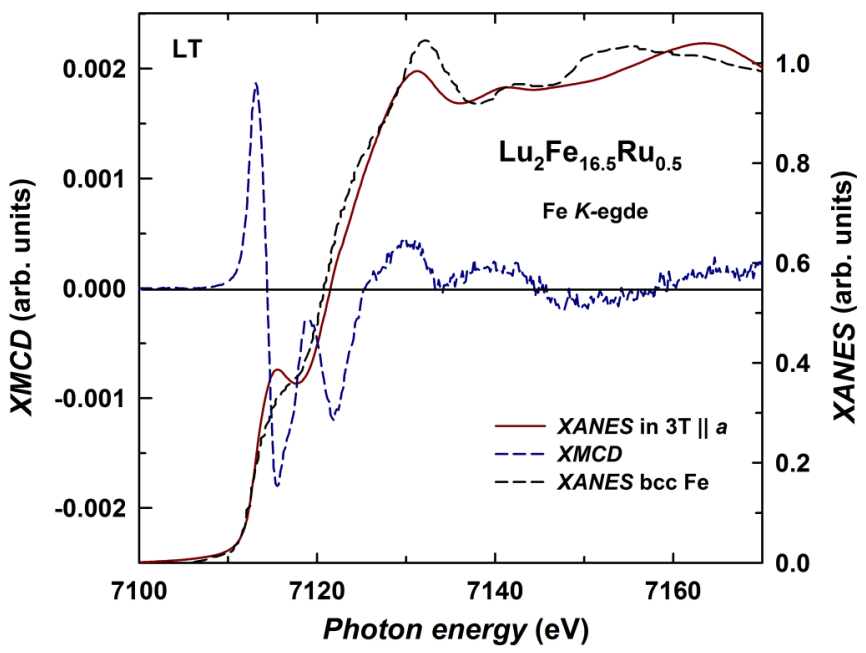

FIG. 3. (Color online) XMCD and XANES signals at the Fe $K$ edge measured on a $\mathrm{Lu}_{2} \mathrm{Fe}_{16.5} \mathrm{Ru}_{0.5}$ single crystal with the magnetic field of $3 \mathrm{~T}$ applied along the easy $a$ axis at $9 \mathrm{~K}$. Adopted from Ref. [30] is the XANES spectrum of Fe foil (bcc Fe) recorded at ambient conditions and matched to fit the experimental data.
Enhancement of the shoulder in $\alpha$-Fe, accompanied by an attenuation of a so-called "white line" and its shift to lower photon energies, has been previously observed by Baudelet et al. [30] in an elemental Fe under pressure, where the structural transition from the bcc to hcp phase takes place above $14 \mathrm{GPa}$. The pre-edge peak in $\mathrm{Lu}_{2} \mathrm{Fe}_{16.5} \mathrm{Ru}_{0.5}$ is similar in shape to that observed in the XANES spectrum of a purely hexagonal Fe phase obtained under the pressure of, e.g., $22.4 \mathrm{GPa}$ [30] and is probably characteristic of $\mathrm{Fe}$ in a hexagonal lattice.

The Fe $K$-edge XMCD spectrum of $\mathrm{Lu}_{2} \mathrm{Fe}_{16.5} \mathrm{Ru}_{0.5}$ is shown in Fig. 3. A main narrow positive peak at the absorption threshold followed by a negative dip of the same amplitude at higher energies is similar to that of elemental iron [30]. The strong positive peak at the absorption edge is usually associated with the presence of unoccupied Fe- $3 d$ states in both spin subbands of $\mathrm{Lu}_{2} \mathrm{Fe}_{16.5} \mathrm{Ru}_{0.5}$. The spectra of $\mathrm{Lu}_{2} \mathrm{Fe}_{16.5} \mathrm{Ru}_{0.5}$ and $\alpha$-Fe begin to differ at energies above $7120 \mathrm{eV}$, where another well-separated negative dip is observed in $\mathrm{Lu}_{2} \mathrm{Fe}_{16.5} \mathrm{Ru}_{0.5}$. This high energy feature was also found in the Fe $K$-edge XMCD spectrum of another " $2: 17$ " compound, $\mathrm{Gd}_{2} \mathrm{Fe}_{17}$ [32]. The difference of the recorded spectrum with the shape of $\mathrm{Fe}$ $K$-edge XMCD of the hydride $\mathrm{Ce}_{2} \mathrm{Fe}_{17}$ [33] may be attributed to the nontrivalent $\mathrm{Ce}$ state of the latter and/or the influence of interstitial hydrogen on the Fe electronic states. These observations allow us to ascribe the recorded signal to the magnetic polarization of the iron sublattice in $\mathrm{Lu}_{2} \mathrm{Fe}_{16.5} \mathrm{Ru}_{0.5}$ only, whereas the rare earth here does not dominate the spectrum, as opposed to the other rare earth $3 d$ metal systems such as, e.g., $\mathrm{ErCo}_{2}$ [34].

The field dependence of the maximum XMCD amplitude obtained at the Fe $K$-edge with the incident energy of $7113 \mathrm{eV}$ is shown in Fig. 4. A corresponding magnetization isotherm of a $\mathrm{Lu}_{2} \mathrm{Fe}_{16.5} \mathrm{Ru}_{0.5}$ single crystal measured along the easy $a$ axis at $9 \mathrm{~K}$ is shown for comparison in the inset to Fig. 5. The

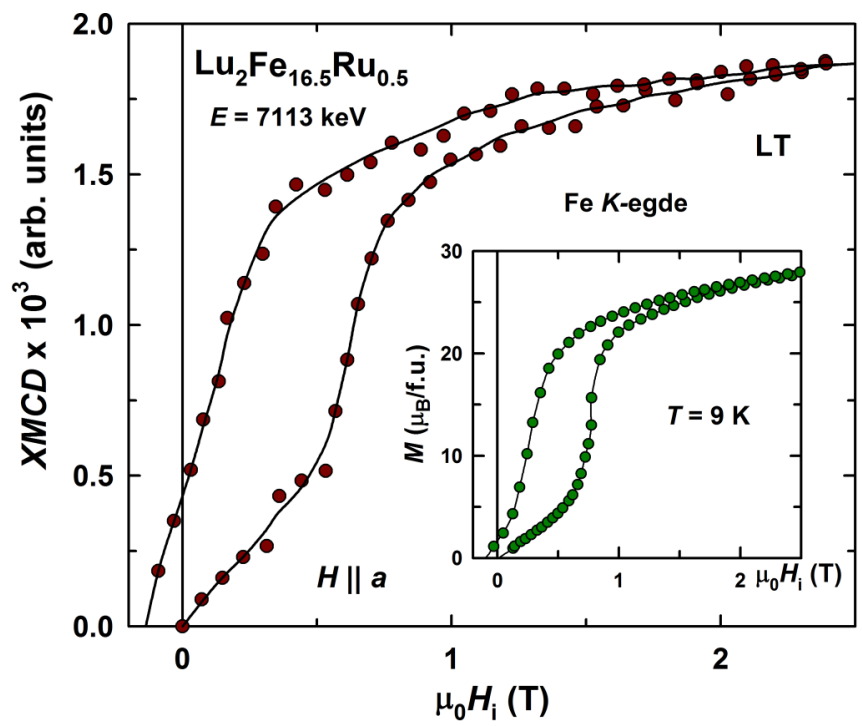

FIG. 4. (Color online) Field dependence of the maximum amplitude of XMCD signal on a Fe $K$ absorption edge measured at the incident energy of $7113 \mathrm{eV}$, with the magnetic field applied along the easy $a$ axis of a $\mathrm{Lu}_{2} \mathrm{Fe}_{16.5} \mathrm{Ru}_{0.5}$ single crystal and a corresponding magnetization isotherm (inset). 


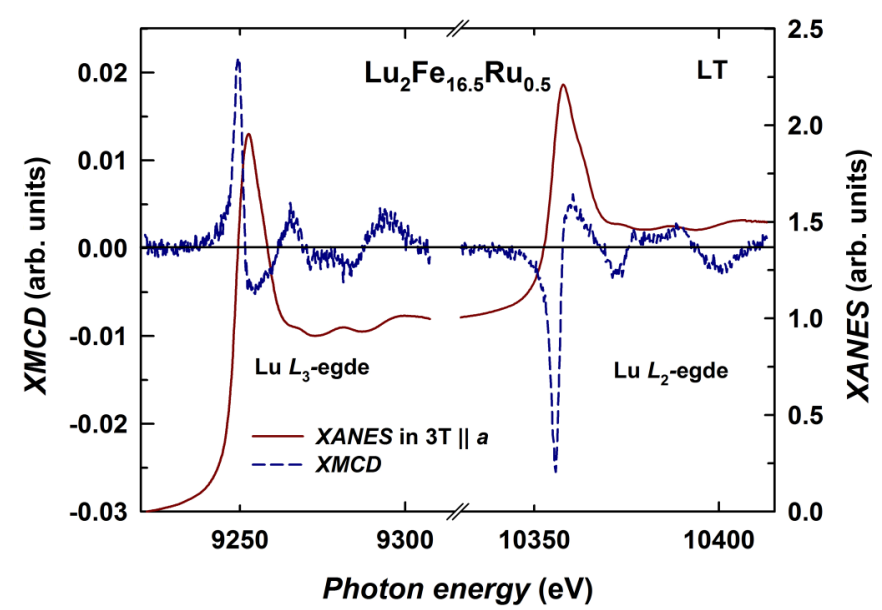

FIG. 5. (Color online) XMCD and XANES signals at the $\mathrm{Lu} L_{3}$ (left) and $L_{2}$ edges (right) measured on a $\mathrm{Lu}_{2} \mathrm{Fe}_{16.5} \mathrm{Ru}_{0.5}$ single crystal with the magnetic field of $3 \mathrm{~T}$ applied along the easy $a$ axis at $9 \mathrm{~K}$.

sample is switched from AF into a noncollinear ferromagnetic state above a field of $0.8 \mathrm{~T}$; the magnetization still increases as it reaches the collinear phase in the direction of the applied field. The Fe-sublattice magnetic behavior in $\mathrm{Lu}_{2} \mathrm{Fe}_{16.5} \mathrm{Ru}_{0.5}$ is consistent with the full magnetization curve. The observed metamagnetic transition is clearly of the first order and exhibits a wide hysteresis.

\section{B. Lutetium $\mathrm{L}_{\mathrm{II}, \mathrm{III}}$ - and ruthenium $\mathrm{L}_{\mathrm{II}}$-edge XANES and XMCD results and analysis}

The XANES spectra of $\mathrm{Lu}_{2} \mathrm{Fe}_{16.5} \mathrm{Ru}_{0.5}$ at the $\mathrm{Lu} L_{3}$ and $L_{2}$ edges are displayed in Fig. 5. (The raw data of the former showed an additional large peak above the absorption edge at $\sim 10375 \mathrm{eV}$ due to diffraction. Because it has not induced any considerable error into the corresponding XMCD signal, it was carefully eliminated prior to presentation.) The white lines at the $L_{2}$ and $L_{3}$ edges of the rare earth elements are related to the transitions from $2 p_{1 / 2}$ and $2 p_{3 / 2}$ to the $5 d_{3 / 2}$ and $5 d_{5 / 2}$ electronic states, respectively. The corresponding XMCD signals are also shown in Fig. 5. The amplitude of the dichroic $L_{2}, 3$ signal is of an order of magnitude larger than that of the $\mathrm{Fe} K$ edge due to a much weaker spin-orbit coupling in the final states of the latter [35].

In the $\mathrm{Lu}-\mathrm{Fe}$ intermetallic compound, $\mathrm{Lu}$ atoms may possess a non-intrinsic, induced magnetic moment originating from the hybridization effects of $5 d$-Lu states with the $3 d$ states of magnetic transition metal matrix (see, e.g., theoretical and experimental results on $\mathrm{LuFe}_{2}[36,37]$ ). Because the $L_{2,3}$ XMCD signals are directly related to the spin polarization of the $d$ final states (and determined by the selection rules) and act as a spin detector of the absorbing atom, the data in Fig. 6 reveal a $5 d$ magnetic moment at the Lu sites in $\mathrm{Lu}_{2} \mathrm{Fe}_{16.5} \mathrm{Ru}_{0.5}$. Left (right) circularly polarized $\mathrm{x}$ rays excite more spin-up (spin-down) electrons from the $2 p_{3 / 2}$ level [35]. The opposite is the case for the $2 p_{1 / 2}$ level. A negative sign of the XMCD $L_{2}$ signal suggests mainly spin-down character of the $5 d$ unoccupied states, while the occupied states must be of a spin-up character. Because the contribution of iron to the magnetism of $\mathrm{Lu}_{2} \mathrm{Fe}_{16.5} \mathrm{Ru}_{0.5}$ is obviously greater than

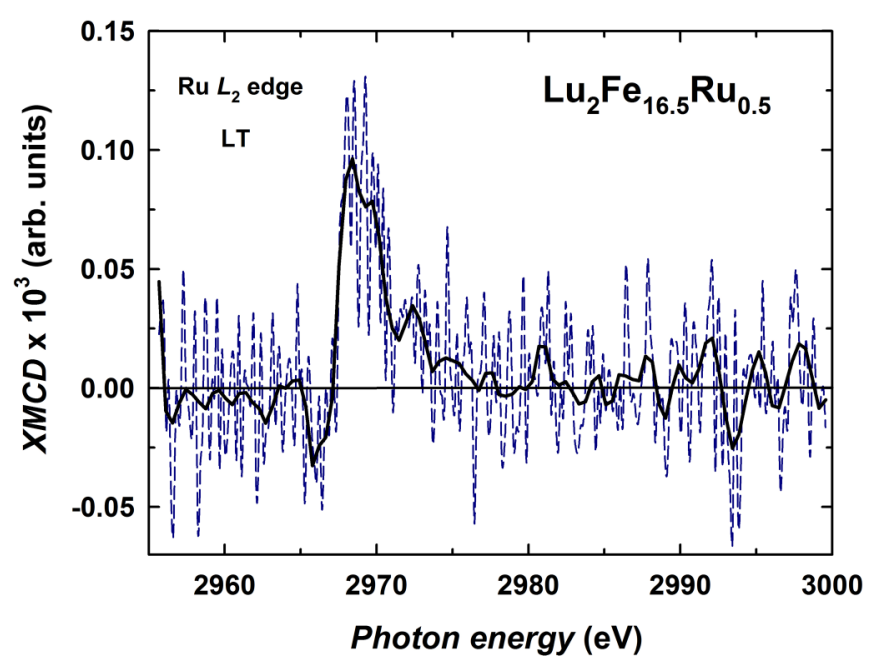

FIG. 6. (Color online) XMCD signal at the $\mathrm{Ru} L_{2}$ edge measured on a $\mathrm{Lu}_{2} \mathrm{Fe}_{16.5} \mathrm{Ru}_{0.5}$ single crystal with the magnetic field of $3 \mathrm{~T}$ applied along the easy $a$ axis at $9 \mathrm{~K}$.

that of $\mathrm{Lu}$, the $\mathrm{Fe}-3 d$ magnetic moments are aligned along the magnetic field direction [or nearly along that in the $3 \mathrm{~T}$ field (cf. Fig. 4)-in this case, the angle $\Theta$ between the magnetic moments and the photon wave vector is rather small, and the XMCD signal would scale as $\left.\cos \Theta \approx 1-\Theta^{2} / 2\right]$. The $\mathrm{Fe}$ $S_{3 d}$ spins therefore lie in the opposite direction. Due to the constant AF interaction between $S_{3 d}$ and $S_{5 d}$ spins [38], the $5 d$ magnetic moment of Lu should align against the applied field, as observed experimentally (Fig. 5).

The intensity ratio between the normalized XMCD amplitudes $L_{2}$ and $L_{3}$ is -1 (or -2 before normalization), which corresponds to the expected branching ratio. (Here, the negative sign is due to opposing spin-orbit coupling of the core states.) Information on the orbital and spin magnetic moments of a given atomic electronic shell can be obtained using sum rules, which involve the integrated intensity of both the absorption spectra and dichroic signal and are independent of the shape of XMCD spectra $[39,40]$,

$$
M_{L}=-2 / 3 \times C \times\left(X_{L 3}+X_{L 2}\right)=0.007 \times C
$$

and

$$
M_{S}=-C \times\left(X_{L 3}-2 X_{L 2}\right)=-0.209 \times C .
$$

Here, $X_{L 3}$ and $X_{L 2}$ are the integrals over the dichroic signal at the $L_{3}$ and $L_{2}$ edges, respectively, and $C$ expressed through the number of holes $n_{h}(=9$ for $\mathrm{Lu})$ as $n_{h} /\left(W_{L 3}+W_{L 2}\right)=0.357$ is a constant. $W_{L 3}$ and $W_{L 2}$ are the integrated intensities of white lines corresponding to the transitions into $5 d$ states of $\mathrm{Lu}$. The analysis provides the following values of orbital and spin moments of Lu $5 d$ states: $M_{L}=0.0025 \mu_{B}$ and $M_{S}=-0.0746 \mu_{B}$, respectively, and the total magnetic moment is $M_{\text {total }}=-0.072 \mu_{B}$.

To shed light on the magnetic polarization of $\mathrm{Ru}$, we planned to measure the XMCD spectra at the $\mathrm{Ru} L_{2}$ and $L_{3}$ (2p-4d dipole transition) absorption edges as a function of photon energy. Unfortunately, the resulting dichroic signal at the $\mathrm{Ru} L_{3}$ edge was undetectable, being comparable with the residual signal. The $\mathrm{Ru} L_{2}$-edge XMCD data measured 
with a magnetic field of $3 \mathrm{~T}$ applied along the easy $a$ axis of $\mathrm{Lu}_{2} \mathrm{Fe}_{16.5} \mathrm{Ru}_{0.5}$ are shown in Fig. 6. Despite a rather distorted signal due to low $\mathrm{Ru}$ content and high overall absorption in $\mathrm{Lu}_{2} \mathrm{Fe}_{16.5} \mathrm{Ru}_{0.5}$ at that energy, the peak observed at $\sim 2970 \mathrm{eV}$ (also inversed by applied magnetic field of the opposite direction) demonstrates the existence of magnetic polarization at the Ru site. Furthermore, the sign of the XMCD signal at the $\mathrm{Ru} L_{2}$ edge suggests ferromagnetic coupling between the $\mathrm{Ru}$ and $\mathrm{Fe}$ moments. To define the local magnetic moments distribution in $\mathrm{Lu}_{2} \mathrm{Fe}_{16.5} \mathrm{Ru}_{0.5}$, we have further employed powder-neutron diffraction.

\section{Neutron-diffraction study}

Temperature evolution of the neutron powder-diffraction profiles from $\mathrm{Lu}_{2} \mathrm{Fe}_{16.5} \mathrm{Ru}_{0.5}$ measured in zero-magnetic field between 285 and $2 \mathrm{~K}$ is shown in Fig. 7. Cooling through the Néel temperature has resulted in the appearance of the superlattice reflections as equally spaced satellites of the allowed nuclear peaks $(101) \pm \tau,(002) \pm \tau$, etc. [where $\tau$ is a wave vector $\left.\tau=\left(0,0, \tau_{z}\right)\right]$ of the $\mathrm{Th}_{2} \mathrm{Ni}_{17}$ structure. The relative intensities of magnetic reflections, including a strong one at the origin of reciprocal lattice $(000)+\tau$, are characteristic of a helical spin configuration. Since no magnetic contribution at the tops of nuclear reflections was detected when varying temperature and only the first-order satellites were observed within the experimental error, the pattern recorded at $2 \mathrm{~K}$ was fitted assuming an appearance of an undistorted AF helix, incommensurate with the crystallographic lattice. In this model, the $c$ axis of the crystal is the screw axis, and the moments are ferromagnetically aligned within the basal planes and rotate from plane to plane with a turn angle determined by the propagation of a wave vector $\tau$ of magnetic structure.

We began the refinement procedure with considering the ideal Fe sublattice (data taken from Table I but with no sites splitting and/or $4 e \mathrm{Fe}$ pairs) and allowed the values of $\mathrm{Fe}$ magnetic moments to vary at different crystallographic sites. As the magnetic moments at $\mathrm{Lu}$ sites in the magnetic cell of $\mathrm{Lu}_{2} \mathrm{Fe}_{16.5} \mathrm{Ru}_{0.5}$, fixed values of $-0.38(-0.36) \mu_{B}$ for $\mathrm{Lu}$ $2 b(2 d)$ obtained from the electronic structure calculations (see Sec. V) were used. Individual refined Fe moments $\mu_{\exp / \text { ideal }}$ are listed in Table II. The neutron-diffraction study was performed in zero-magnetic field. The magnetization data in Fig. 4 qualitatively demonstrates the effect of applying a magnetic field in the direction perpendicular to the helix axis. It first distorts the helix by giving rise to a magnetic moment along the field, and at a certain critical field $(<1 \mathrm{~T})$, a first-order phase transition to a fan structure takes place. A further increase in the magnetic field continuously reduces the opening angle of the fan. The values of all local magnetic moments in case of an ideal structural model would sum into a saturation magnetization of $31.2 \mu_{B} /$ f.u. (ferrimagnetic alignment of $\mathrm{Lu}$ and Fe magnetic moments is taken into account), whereas the magnetization data from Ref. [26] obtained on a single crystal provide a value of $\sim 34 \mu_{B} /$ f.u. in the $14 \mathrm{~T}$ magnetic field. The fit quality for the diffraction pattern in this case was essentially rather poor.

Increasing the disorder in the crystal structure via introducing the $4 e \mathrm{Fe}$ sites was found to complicate the magnetic moments determination. Reasonable compromise was achieved while keeping the magnetic moments at the $4 f$

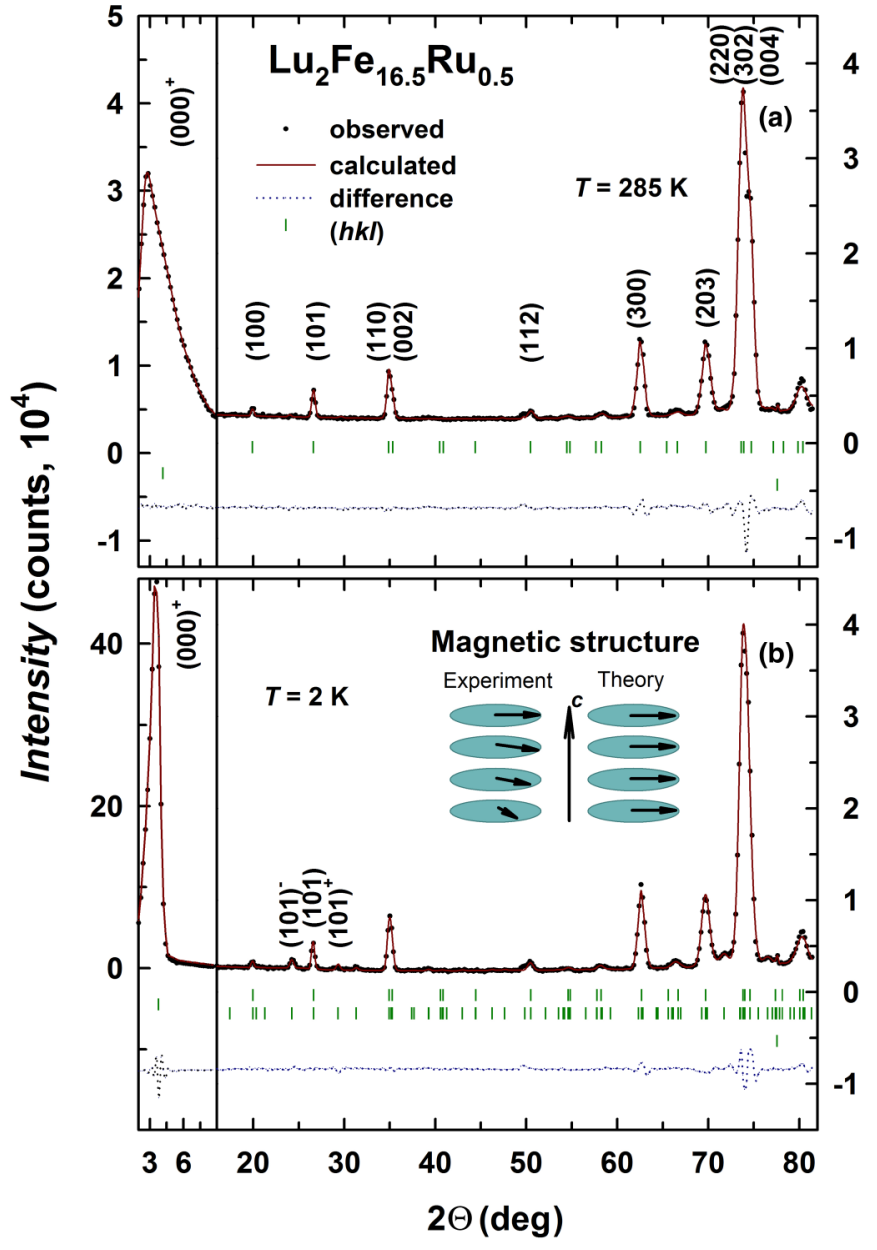

FIG. 7. (Color online) Parts of the powder neutron-diffraction profiles ( $\lambda=2.52 \AA$ ) from $\mathrm{Lu}_{2} \mathrm{Fe}_{16.5} \mathrm{Ru}_{0.5}$ at selected temperatures, with the results of fitting by the Rietveld method. The diamonds are the observed data, the solid lines are the calculated patterns, and the dotted line shows their difference. Positions of Bragg reflections for the main phase and a minor $\alpha$-Fe contaminant for each temperature are marked together with the magnetic structure data. The agreement factors of the fit are at $285 \mathrm{~K} \chi^{2}=10.2\left(R_{\exp }=3.3\right), R_{\text {Bragg }}=2.1(\%)$; at $2 \mathrm{~K} \chi^{2}=9.7\left(R_{\exp }=3.3\right), R_{\text {Bragg }}=1.5$, magnetic $R_{\mathrm{M}}=10.6(\%)$. The inset in Fig. 7(b) displays the helical spin model used for fitting the experimental data and the ferromagnetic configuration predicted by theoretical calculations (see Sec. V for details).

and $4 e$ positions constrained to be equal during the refinement. This procedure was necessary due to a small percentage of the latter and was justified by similitude between the $4 e$ and $4 f \mathrm{Fe}$ positions, which both correspond to the Fe-Fe pairs substituted for Lu. Moreover, Mössbauer effect studies of $R_{2} \mathrm{Fe}_{17}$ support this hypothesis by reporting similar hyperfine fields at the $4 e$ and $4 f \mathrm{Fe}$ sites, being far the largest among those at various crystallographic positions in the $R_{2} \mathrm{Fe}_{17}$ compounds [41]. The magnetic moments at the majority of Fe sites increase with the increase of disorder in the crystal structure. For $24 \%$ of $4 e$ Fe dumbbell pairs, $\mu_{4 f}$ remained almost unchanged within the experimental uncertainty (Table II). The fit quality was improved considerably and has led to the total magnetization value of $34.6 \mu_{B} /$ f.u. The pattern and the best fit are shown in Fig. 7(b). 
TABLE II. Experimental site-resolved magnetic moments $\mu_{\exp }$ of $\mathrm{Lu}_{2} \mathrm{Fe}_{16.5} \mathrm{Ru}_{0.5}$ at $2 \mathrm{~K}$ from the neutron powder-diffraction experiment obtained for the ideal ( $0 \% 4 e \mathrm{Fe}$ atoms) and real (24\% $4 e \mathrm{Fe}$ atoms) crystal structure models and helical spin configuration. Indicators of the fit quality are listed as well.

\begin{tabular}{lccc}
\hline \hline Atom & Site & $\mu_{\text {exp } / \text { ideal }}\left(\mu_{\mathrm{B}}\right)$ & $\mu_{\text {exp } / \text { real }}\left(\mu_{\mathrm{B}}\right)$ \\
\hline $\mathrm{Lu}_{1}$ & $2 b$ & -0.38 & -0.38 \\
$\mathrm{Lu}_{2}$ & $2 d$ & -0.36 & -0.36 \\
$\mathrm{Fe}_{1}$ & $4 f$ & $2.4(0.1)$ & $2.3(0.1)$ \\
$\mathrm{Fe}_{2}$ & $6 g$ & $1.8(0.2)$ & $1.9(0.2)$ \\
$\mathrm{Fe}_{3(1,2)}$ & $12 j$ & $2.1(0.1)$ & $2.2(0.1)$ \\
$\mathrm{Fe}_{4(1,2)}$ & $12 k$ & $1.7(0.1)$ & $2.0(0.1)$ \\
$\mathrm{Ru}_{4(2)}$ & $12 k$ & - & - \\
$\mathrm{Fe}_{5}$ & $4 e$ & - & $2.3(0.1)$ \\
$R_{\mathrm{Bragg}}(\%)$ & & 3.7 & 1.5 \\
$R_{\mathrm{F}}(\%)$ & & 3.7 & 1.6 \\
$R_{\mathrm{M}}(\%)$ & & 17.6 & 9.6 \\
$\chi^{2}$ & & 13.6 & 9.7 \\
\hline \hline
\end{tabular}

The propagation vector of the helical structure was found to vary from $0.236 c^{*}$ directly below $T_{\mathrm{N}}$ down to $0.219 c^{*}$ at $2 \mathrm{~K}$. At low temperatures, the magnetic moments within one plane turn from the initial direction by $2 \pi \tau \approx 79^{\circ}$ over one lattice constant along the $c$ axis; therefore, the magnetic spiral along the $c$ axis repeats itself every 4.5 crystallographic unit cells [for schematic representation of the magnetic helix, see Fig. 7(b)]. A rather small and broad low angle (000) ${ }^{+}$peak still observed at near room temperature [Fig. 8(a)] is probably due to a short range magnetic order present in the paramagnetic state of $\mathrm{Lu}_{2} \mathrm{Fe}_{16.5} \mathrm{Ru}_{0.5}$.

An attempt to independently refine the values of induced magnetic moments at Lu has not brought any luck due to (i) already complex crystal and magnetic structures and (ii) broad spatial distribution of $5 d$ electrons. The latter causes the magnetic form factor of $\mathrm{Lu}$ to decrease rapidly with the neutron-scattering vector, thus decreasing the experimental sensitivity for the precise magnetic moment determination. A possible cause for the small but noticeable asymmetry in " $+\tau$ " and " $-\tau$ " magnetic satellites is a minor deviation from a simple spiral model due to the preferential substitution of $\mathrm{Ru}$ for Fe at a certain lattice site. However, a small amount of $\mathrm{Ru}$ and a typical precision of the powder neutron-diffraction technique did not allow us to confirm the occurrence of magnetic polarization at $\mathrm{Ru}$ and/or to quantify possible phase difference (if any) for $\mathrm{Fe}$ and $\mathrm{Ru}$ spins in the unit cell.

\section{ELECTRONIC-STRUCTURE CALCULATIONS}

To examine theoretically the electronic structure of $\mathrm{Lu}_{2} \mathrm{Fe}_{16.5} \mathrm{Ru}_{0.5}$ and to make a comparison with experimental data, we performed first-principles calculations based on the local spin density approximation (LSDA) [42]. For this purpose, we employed a Korringa-Kohn-Rostoker Green Function-based method with the atomic sphere approximation (KKR-ASA), as described in Ref. [43]. Spherical harmonic expansion up to $l_{\max }=3$ (spdf-basis set) and set of $30 \mathrm{k}$-points in an irreducible wedge of a Brillouin zone were used to achieve convergence. The sizes of the ASA sphere radii were chosen as $R(\mathrm{Lu}) / R(\mathrm{Fe})=1.4$. The $\mathrm{Th}_{2} \mathrm{Ni}_{17}$ structure

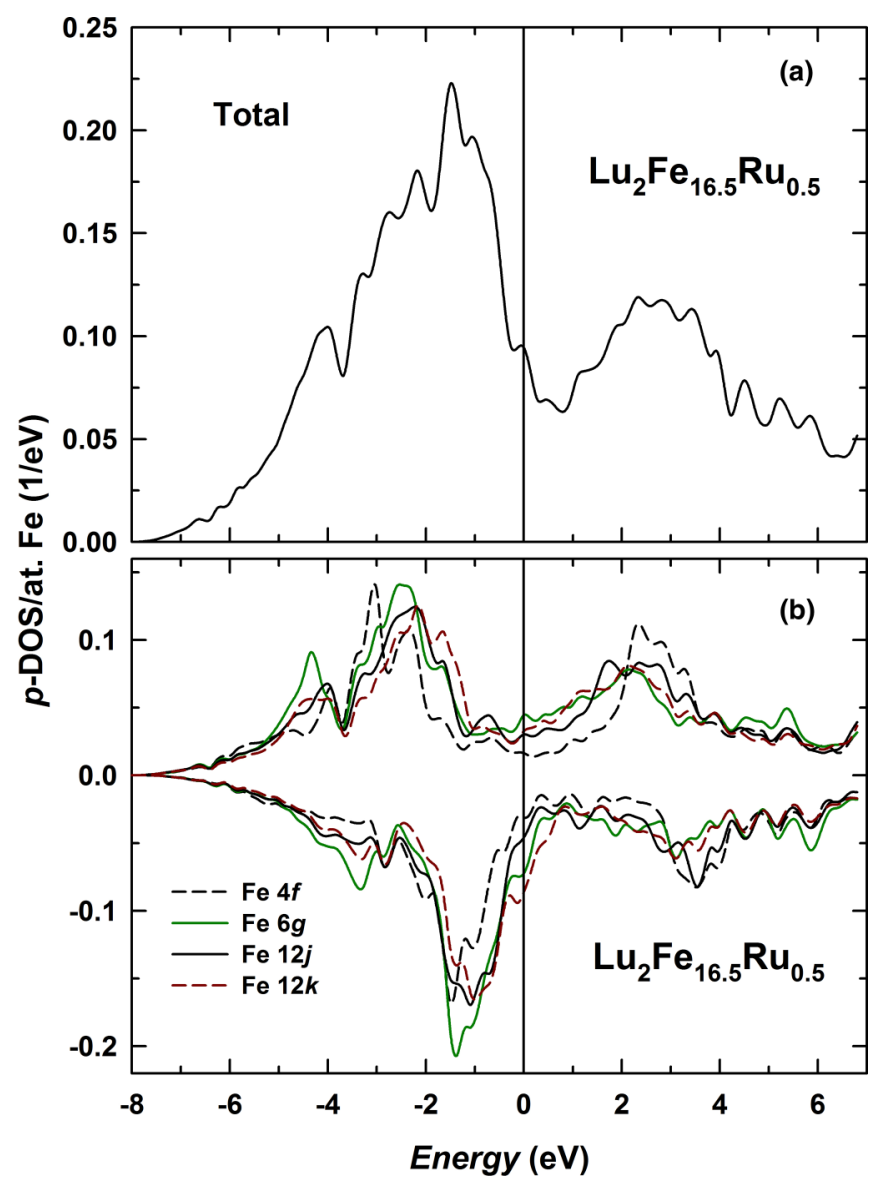

FIG. 8. (Color online) (a) The total projected electron-energy density of $p$-orbital states ( $p$-DOS) of $\mathrm{Fe}$ atoms in $\mathrm{Lu}_{2} \mathrm{Fe}_{16.5} \mathrm{Ru}_{0.5}$ given per atom from LSDA calculations. (b) The $p$-orbital projected DOS for Fe atoms at $4 f, 6 g, 12 j$, and $12 k$ special positions of the $P 6_{3} / m m c$ space group.

model that included no lattice-site splitting and/or additional $4 e \mathrm{Fe}$ atomic pairs (that is, perfect) has been used for the calculations, and principal atomic positions were taken from Table I as fully populated. The advantage of this approach is that it allows us to discern the structural disorder effects and magnetism in the system. The atomic disorder effects due to the $\mathrm{Ru}$ substitution at the $12 k$ Fe sites were treated within the conventional coherent potential approximation (CPA) [44].

Surprisingly, the ground state of $\mathrm{Lu}_{2} \mathrm{Fe}_{16.5} \mathrm{Ru}_{0.5}$ calculated in this way is ferromagnetic. The total projected density of $p$-orbital states ( $p$-DOS) of Fe atoms obtained as a sum over four Fe sites in $\mathrm{Lu}_{2} \mathrm{Fe}_{16.5} \mathrm{Ru}_{0.5}$ is shown in Fig. 8(a). The site-resolved $p$-orbital states of $\mathrm{Lu}_{2} \mathrm{Fe}_{16.5} \mathrm{Ru}_{0.5}$ are presented in Fig. 8(b). The Fermi energy $E_{\mathrm{F}}$ in all figures is set at 0 . Presence of Fe- $3 d$ states in both spin sub-bands of $\mathrm{Lu}_{2} \mathrm{Fe}_{16.5} \mathrm{Ru}_{0.5}$ agrees with the measured Fe $K$-edge XMCD spectrum in Fig. 3. The main peak of the minority spin band is centered at around $-2 \mathrm{eV}$ to $E_{\mathrm{F}}$, whereas the majority spin band is mainly contributing to the DOS below $-2 \mathrm{eV}$. A small DOS peak in the vicinity of $E_{\mathrm{F}}$ is mostly of a spin-down character and is caused by the $12 k$ and $6 g$ atomic Fe positions (i.e., those in close proximity with $\mathrm{Ru}$ atoms), both having sharp edges in the $p$-DOS at the Fermi energy. 
TABLE III. Calculated site-resolved magnetic moments (in $\mu_{B}$ /atom) of (i) $\mathrm{Lu}_{2} \mathrm{Fe}_{16.5} \mathrm{Ru}_{0.5}$ having an ideal crystal structure (no lattice site splitting and/or additional $4 e$ Fe atomic pairs) with principal atomic positions taken from Table I as fully populated and (ii) a reference hypothetic $\mathrm{Lu}_{2} \mathrm{Fe}_{17}$ * compound with the structural data as above.

\begin{tabular}{lccccccc}
\hline \hline Compound & $\mathrm{Fe}, 12 k$ & $\mathrm{Fe}, 12 j$ & $\mathrm{Fe}, 6 g$ & $\mathrm{Fe}, 4 f$ & $\mathrm{Lu}, 2 b$ & $\mathrm{Lu}, 2 d$ & $\mathrm{Ru}, 12 k$ \\
\hline $\mathrm{Lu}_{2} \mathrm{Fe}_{16.5} \mathrm{Ru}_{0.5}$ & 1.89 & 2.07 & 1.55 & 2.50 & -0.38 & -0.36 \\
$\mathrm{Lu}_{2} \mathrm{Fe}_{17} *$ & 1.86 & 2.08 & 1.51 & 2.52 & -0.39 & -0.38 & - \\
\hline \hline
\end{tabular}

Table II reports the calculated individual magnetic moments at various lattice sites in $\mathrm{Lu}_{2} \mathrm{Fe}_{16.5} \mathrm{Ru}_{0.5}$. As an overall assessment, the sequence of magnetic moments $\mu_{\mathrm{Fe} 4 f}>\mu_{\mathrm{Fe} 12 j}$ $>\mu_{\mathrm{Fe} 12 k}>\mu_{\mathrm{Fe} 6 g}$ is in agreement with the neutron-diffraction experiment and is due to different volumes and the number of nearest neighbors for each of four inequivalent $\mathrm{Fe}$ sites in the hexagonal $\mathrm{Th}_{2} \mathrm{Ni}_{17}$ crystal structure. The calculated magnetic moments induced at $\mathrm{Lu} 2 b$ and $2 d$ sites are -0.38 and $-0.36 \mu_{B}$ /at., respectively, being close in value to those obtained for another iron-rich phase, $\mathrm{LuFe}_{2}$ [36,37]. A smaller average Lu moment is derived from the XMCD data at the maximum applied magnetic field of $3 \mathrm{~T}, M_{\mathrm{Lu}}=-0.072 \mu_{B}$. Since the magnetization does not reach full saturation at this field (see Fig. 4), it is natural to assume that the increase of the field would increase the magnetic moments of both $\mathrm{Fe}$ and Lu. Both theoretical and experimental XMCD data nevertheless yield the same coupling scheme for the Lu $5 d$ - and Fe $3 d$-magnetic moments. Further, the calculations provide an induced magnetic moment of $0.06 \mu_{B}$ /at. at Ru coupled ferromagnetically with Fe. The total magnetization $33.03 \mu_{B} /$ f.u. is in good agreement with the experimental values both for the field-induced ferromagnetic state in $\mathrm{Lu}_{2} \mathrm{Fe}_{16.5} \mathrm{Ru}_{0.5}$ [26] and obtained as a sum of magnetic moments derived from the neutron-diffraction experiment, $34 \mu_{B} /$ f.u. and $34.6 \mu_{B} /$ f.u., respectively.

Only weak electronic structure changes in the Ru-undoped compound, $\mathrm{Lu}_{2} \mathrm{Fe}_{17}$ * (a hypothetical reference material with the same structural characteristics as $\mathrm{Lu}_{2} \mathrm{Fe}_{16.5} \mathrm{Ru}_{0.5}$ with $\mathrm{Ru}$ content being nil) are detected. The calculations show that $\mathrm{Lu}_{2} \mathrm{Fe}_{17}$ * orders ferromagnetically. As seen from Table III, absence of $\mathrm{Ru}$ in the $12 k-6 g$ kagome nets slightly decreases the magnetic moments of $\mathrm{Fe}$ atoms at these positions and increases marginally the moments at the neighboring $12 j$ and $4 f \mathrm{Fe}$ sites. The total magnetic moment of $\mathrm{Lu}_{2} \mathrm{Fe}_{17}$ * is $32.44 \mu_{B} /$ f.u.

In order to explore further the role of $\mathrm{Ru}$ on magnetic interactions in $\mathrm{Lu}_{2} \mathrm{Fe}_{16.5} \mathrm{Ru}_{0.5}$, we have estimated the effective exchange in the system by employing Liechtenstein Green Function formalism based on magnetic force theorem [45]. In the particular implementation used here [46], a total effective exchange experienced by a given atomic site in the ferromagnetic state (so-called $J_{0}$ constant) was calculated. This effective exchange was defined as a sum of all distant pair-wise exchange interactions of a given atomic moment with the rest of magnetic sites. Namely, the sum $J_{0}=\sum_{i} J_{0 i}$ was calculated, with $J_{i j}$ as a parameter of the model classical Heisenberg Hamiltonian

$$
H=\sum_{i j} J_{i j} \vec{e}_{i} \vec{e}_{j},
$$

where $\vec{e}_{i}$ is the unit vector of a spin at the $i$ th lattice site. The results of $J_{0}$ calculations are presented in Table IV. In contrast to the common speculation on AF interaction in the $4 f$ dumbbell pairs of $R_{2} \mathrm{Fe}_{17}$ compounds [11], we find strong ferromagnetic exchange between the $4 f \mathrm{Fe}$ atoms in $\mathrm{Lu}_{2} \mathrm{Fe}_{16.5} \mathrm{Ru}_{0.5}$. In addition, $\mathrm{Ru}$ doping seems to affect the exchange coupling stronger than the magnitudes of individual Fe moments. The absolute $J_{0}$ values in $\mathrm{Lu}_{2} \mathrm{Fe}_{16.5} \mathrm{Ru}_{0.5}$ increase for $4-20 \%$ for various Fe lattice sites as compared to $\mathrm{Lu}_{2} \mathrm{Fe}_{17}$ * The maximum increase corresponds to the $12 k$ Fe moments, whereas the minimum is found for the $4 f$ sites. As an overall conclusion, $\mathrm{Ru}$ should strengthen the ferromagnetic exchange in the ideal system.

\section{DISCUSSION AND CONCLUSIONS}

Despite an overall good quantitative agreement on the values of magnetic moments at the three constituent elements in $\mathrm{Lu}_{2} \mathrm{Fe}_{16.5} \mathrm{Ru}_{0.5}$ obtained theoretically and experimentally, the major discrepancy is observed in the type of magnetic ordering of the compound. The first-principle KKR CPA calculations show that a partial replacement of $\mathrm{Fe}$ by $\mathrm{Ru}$ atoms in a fixed crystal structure should reinforce the ferromagnetic exchange. Furthermore, we find that all pair-wise exchange interactions in the ideal $\mathrm{Lu}_{2} \mathrm{Fe}_{16.5} \mathrm{Ru}_{0.5}$ crystal are positive, with the strongest exchange in the $4 f$ dumbbell Fe pairs of approximately $54 \mathrm{meV}$ (cf. with $20.6 \mathrm{meV}$ obtained theoretically for the same positions in the hexagonal, ferromagnetic $\mathrm{Gd}_{2} \mathrm{Fe}_{17}$ [47]). The deviation of theoretical calculations from the experimentally observed spiral-spin configuration seemingly stems from simulating a perfect crystal, while experimental data are obtained on an off-stoichiometric compound with local structural defects.

Interestingly enough, similar influence of $\mathrm{Ru}$ doping on magnetism of $\mathrm{Y}_{2} \mathrm{Fe}_{17}$ was reported in Ref. [48]. The single-crystalline neutron-diffraction study on a hexagonal $\mathrm{Y}_{2} \mathrm{Fe}_{17}$ [49] has shown that the compound is almost stoichiometric despite the structural defects. According to our present

TABLE IV. Exchange constants $J_{0}$ (in $\mathrm{meV}$ ) calculated for the same cases as in Table III.

\begin{tabular}{lcccccc}
\hline \hline Compound & $\mathrm{Fe}, 12 k$ & $\mathrm{Fe}, 12 j$ & $\mathrm{Fe}, 6 g$ & $\mathrm{Fe}, 4 f$ & $\mathrm{Lu}, 2 b$ & $\mathrm{Lu}, 2 d$ \\
\hline $\mathrm{Lu}_{2} \mathrm{Fe}_{16.5} \mathrm{Ru}_{0.5}$ & 41.23 & 69.39 & 64.08 & 82.59 & -17.14 & -15.51 \\
$\mathrm{Lu}_{2} \mathrm{Fe}_{17}{ }^{*}$ & 34.42 & 62.18 & 55.92 & 79.32 & -18.23 & -17.55 \\
\hline \hline
\end{tabular}


calculations, only weak electronic structure changes due to the substitution of a few percent of $\mathrm{Fe}$ atoms by $\mathrm{Ru}$ atoms are induced in $\mathrm{Lu}_{2} \mathrm{Fe}_{17}$ with the ideal $\mathrm{Th}_{2} \mathrm{Ni}_{17}$ structure, and those are not sufficient to promote the magnetic order change in the material. Careful inspection of Fig. 4 also reveals a small remanence in $\mathrm{Lu}_{2} \mathrm{Fe}_{16.5} \mathrm{Ru}_{0.5}$ after the magnetic field has been fully removed. This weak residual ferromagnetism seems to be intrinsic to both $\mathrm{Ru}$-doped $\mathrm{Lu}_{2} \mathrm{Fe}_{17}$ and $\mathrm{Y}_{2} \mathrm{Fe}_{17}$. Moreover, it cannot be explained by the presence of a ferromagnetic impurity in both compounds, as the remanence gradually decreases until it has ceased at temperatures above $40 \mathrm{~K}$ [48]. The temperature is too low to be ascribed to any Fe-containing substance.

Weak ferromagnetism in some AF systems has been shown to appear as a consequence of pair-wise DzyaloshinskiiMoriya (DM) spin interactions [50-52]. In nonsymmetric $\mathrm{FeGe}$, for instance, the DM interaction is shown to be responsible for the long-period magnetic spiral structure [53]. Local structural inhomogeneities lower the symmetry of the individual $\mathrm{Fe}$ atomic sites in the hexagonal $\mathrm{Th}_{2} \mathrm{Ni}_{17}$ lattice of $\mathrm{Lu}_{2} \mathrm{Fe}_{16.5} \mathrm{Ru}_{0.5}$ and, in principle, may favor the onset of these interactions at some of the Fe-Fe bonds. The magnetic helix in $\mathrm{Lu}_{2} \mathrm{Fe}_{16.5} \mathrm{Ru}_{0.5}$ observed at $2 \mathrm{~K}$ in zero magnetic field is characterized by a period of 4.5 unit cells, i.e., it involves $19 \mathrm{Fe}$ layers. It should be noted that the interactions causing the AF cone structure in $\mathrm{Lu}_{2} \mathrm{Fe}_{16.5} \mathrm{Ru}_{0.5}$ must not be too strong: the spin-spiral structure in the basal plane is destroyed by the magnetic field of less than $1 \mathrm{~T}$ (see Fig. 4). Although modelling the DM interactions in such a complex structure as $\mathrm{Lu}_{2} \mathrm{Fe}_{16.5} \mathrm{Ru}_{0.5}$ can be difficult, the hypothesis concerning relativistic origin of the spin spiral in this material is worthy of attention and further elucidations.

We find that $\mathrm{Lu}_{2} \mathrm{Fe}_{16.5} \mathrm{Ru}_{0.5}$ displays an Invar-like behavior in a wide temperature range reflected in an anomalous temperature variation of lattice parameters (detailed study of magnetoelasticity of $\mathrm{Lu}_{2} \mathrm{Fe}_{16.5} \mathrm{Ru}_{0.5}$ will be presented elsewhere). This suggests that the interplay between local lattice distortions and magnetovolume effects is rather complex. Spontaneous volume strain proportional to the square of magnetization [12] most likely increases stress locally through the varied individual magnetic moments across the lattice sites. This can be a driving force toward strengthening the overall AF exchange in the $\mathrm{Ru}$-doped $\mathrm{Lu}_{2} \mathrm{Fe}_{17}$.

To conclude, we have performed an element-specific probing of local magnetic polarization at each of three chemical species of a single-crystalline $\mathrm{Lu}_{2} \mathrm{Fe}_{16.5} \mathrm{Ru}_{0.5}$ by hard $\mathrm{x}$-ray absorption spectroscopy technique. Lutetium and ruthenium are shown to carry a noticeable magnetic polarization antiparallel and parallel to the dominant Fe sublattice magnetization, respectively. Neutron powder-diffraction experiment revealed a preferential occupation of the $12 k$ sites by $\mathrm{Ru}$ in the partially disordered $\mathrm{Th}_{2} \mathrm{Ni}_{17}$ type of crystal structure. Below the ordering temperature of $208 \mathrm{~K}$, neutron-diffraction patterns were fitted using a spiral spin model. The first-principle KKR CPA calculations performed with the use of an ideal structure model provide the values of site-resolved magnetic moments close to those found experimentally. The calculations, however, suggest that $\mathrm{Lu}_{2} \mathrm{Fe}_{16.5} \mathrm{Ru}_{0.5}$ is a ferromagnet-a result that is in conflict with the observed stabilization of the helical AF configuration. In contrast to relatively negligible electronic effects due to the isoelectronic substitution of a few percent of $\mathrm{Ru}$ atoms for $\mathrm{Fe}$, local structural inhomogeneities and distortions most likely have the strongest impact on exchange interactions, thus defining the final magnetic state of the compound. Relativistic cause of the spin spiral in $\mathrm{Lu}_{2} \mathrm{Fe}_{16.5} \mathrm{Ru}_{0.5}$ cannot be fully excluded.

\section{ACKNOWLEDGMENTS}

The work has been supported by the Czech Science Foundation (Grant No. P204/12/0150). The authors thank the Institut Laue Langevin for the access to the neutron facility. The sample preparation and magnetization measurements have been performed in MLTL (http://mltl.eu) and supported within the program of Czech Research Infrastructures (Project No. LM2011025). S.K. acknowledges the support of the Austrian Science Fund (FWF) (SFB ViCoM F4109-N13).
[1] M. F. Collins and G. G. Low, Proc. Phys. Soc. 86, 535 (1965).

[2] G. Shirane, R. Nathans, and C. W. Chen, Phys. Rev. 134, A1547 (1964).

[3] G. R. Harp, S. S. P. Parkin, W. L. O’Brien, and B. P. Tonner, Phys. Rev. B 51, 12037 (1995).

[4] I. A. Campbell, Proc. Phys. Soc. 89, 71 (1966).

[5] B. Drittler, N. Stefanou, S. Blügel, R. Zeller, and P. H. Dederichs, Phys. Rev. B 40, 8203 (1989).

[6] K. Nakamura, R. Arita, and H. Ikeda, Phys. Rev. B 83, 144512 (2011); M. Tropeano, M. R. Cimberle, C. Ferdeghini, G. Lamura, A. Martinelli, A. Palenzona, I. Pallecchi, A. Sala, I. Sheikin, F. Bernardini, M. Monni, S. Massidda, and M. Putti, ibid. 81, 184504 (2010).

[7] R. S. Dhaka, C. Liu, R. M. Fernandes, R. Jiang, C. P. Strehlow, T. Kondo, A. Thaler, J. Schmalian, S. L. Bud'ko, P. C. Canfield, and A. Kaminski, Phys. Rev. Lett. 107, 267002 (2011).
[8] J. J. M. Franse and R. J. Radwanski, in Handbook of Magnetic Materials, edited by K. H. J. Buschow (Elsevier, Amsterdam, 1993), Vol. 7, p. 307.

[9] A. E. Clark, in Handbook of Ferromagnetic Materials, edited by E. P. Wohlfarth (Elsevier, Amsterdam, 1980), Vol. 1, p. 531.

[10] R. F. Sabiryanov and S. S. Jaswal, Phys. Rev. B 57, 7767 (1998).

[11] D. Givord and R. Lemaire, IEEE Trans. Magn. 10, 109 (1974).

[12] A. V. Andreev, in Handbook of Magnetic Materials, edited by K. H. J. Buschow (Elsevier, Amsterdam, 1995), Vol. 8, p. 59.

[13] R. Plumier and M. Sougi, Proceedings of ICM 1973 (Nauka, Moscow) 3, 487 (1974); Y. Janssen, S. Chang, A. Kreyssig, A. Kracher, Y. Mozharivskyj, S. Misra, and P. C. Canfield, Phys. Rev. B 76, 054420 (2007). 
[14] D. Givord and R. Lemaire, C. R. Acad. Sci. (Paris) 274, 1166 (1972).

[15] S. A. Nikitin, A. M. Tishin, M. D. Kuz'min, and Yu. I. Spichkin, Phys. Lett. A 153, 155 (1991); O. Prokhnenko, J. Kamarad, K. Prokes, Z. Arnold, and A. V. Andreev, Phys. Rev. Lett. 94, 107201 (2005).

[16] A. S. Andreenko, S. A. Nikitin, and Yu. I. Spichkin, Sov. Phys. Solid State 34, 972 (1992).

[17] D. Givord, R. Lemaire, J. M. Moreau, and E. Roudaut, J. Less Comm. Met. 29, 361 (1972).

[18] J. Kamarád, M. Mišek, K. Prokeš, S. Mat’aš, and Z. Arnold, J. Phys. Conf. Series 340, 012067 (2012).

[19] O. Isnard, S. Miraglia, J. L. Soubeyroux, D. Fruchart, and P. l'Héritier, J. Magn. Magn. Mater. 137, 151 (1994).

[20] E. A. Tereshina, H. Yoshida, A. V. Andreev, I. S. Tereshina, K. Koyama, and T. Kanomata, J. Phys. Soc. Japan 76, 82 (2007).

[21] V. I. Voronin, I. F. Berger, and A. G. Kuchin, Phys. Met. Metallogr. 93, 429 (2002).

[22] A. G. Kuchin, W. Iwasieczko, H. Drulis, and V. I. Khrabrov, Solid State Comm. 146, 446 (2008).

[23] J. Kamarad, A. V. Andreev, Z. Machatova, and Z. Arnold, J. Alloys Comp. 408-412, 151 (2006).

[24] E. A. Tereshina, A. V. Andreev, H. Drulis, and S. Daniš, IEEE Trans. Magn. 44, 4210 (2008).

[25] E. A. Tereshina, A. V. Andreev, J. Kamarad, and O. Isnard, J. Appl. Phys. 105, 07A747 (2009).

[26] E. A. Tereshina and A. V. Andreev, Intermetallics 18, 1205 (2010).

[27] D. I. C. Pearson and J. M. Williams, J. Phys. F: Met. Phys. 9, 1797 (1979).

[28] J. Goulon, C. Goulon-Ginet, R. Cortes, and J. M. Dubois, J. Phys. (Paris) 43, 539 (1982); L. Troger, D. Arvanitis, K. Baberschke, H. Michaelis, U. Grimm, and E. Zschech, Phys. Rev. B 46, 3283 (1992); P. Pfalzer, J.-P. Urbach, M. Klemm, S. Horn, M. L. denBoer, A. I. Frenkel, and J. P. Kirkland, ibid. 60, 9335 (1999).

[29] A. V. Andreev, K. Koyama, E. A. Tereshina, J. Prokleška, and K. Watanabe, J. Alloys Comp. 477, 62 (2009).

[30] F. Baudelet, S. Pascarelli, O. Mathon, J. P. Itie, A. Polian, M. d'Astuto, and J. C. Chervin, J. Phys. Condens. Matter 17, S957 (2005).

[31] A. Bianconi, in X-ray Absorption: Principles, Applications, Techniques of EXAFS, SEXAFS, and XANES, edited by D. C. Koningsberger and R. Prins (Wiley, New York, 1988), Ch. 11.
[32] F. Baudelet, C. Brouder, E. Dartyge, A. Fontaine, J. P. Kappler, and G. Krill, J. Magn. Magn. Mater. 104-107, 1418 (1992).

[33] O. Isnard, S. Miraglia, D. Fruchart, C. Giorgetti, S. Pizzini, E. Dartyge, G. Krill, and J. P. Kappler, Phys. Rev. B 49, 15692 (1994).

[34] J. Herrero-Albillos, D. Paudyal, F. Bartolome, L. M. Garcia, V. K. Pecharsky, K. A. Gschneidner Jr., A. T. Young, N. Jaouen, and A. Rogalev, J. Appl. Phys. 103, 07E146 (2008).

[35] C. Brouder and M. Hikam, Phys. Rev. B 43, 3809 (1991).

[36] M. S. S. Brooks, L. Nordstrom, and B. Johansson, J. Phys. Condensed Matter 1, 5861 (1989); Ch. Zhang, Zh. Zhang, Sh. Wang, H. Li, J. Dong, N. Xing, Y. Guo, and W. Li, Solid State Comm. 142, 477 (2007).

[37] C. Giorgetti, S. Pizzini, E. Dartyge, A. Fontaine, F. Baudelet, C. Brouder, Ph. Bauer, G. Krill, S. Miraglia, D. Fruchart, and J. P. Kappler, Phys. Rev. B 48, 12732 (1993); A. P. Murani, Physica B Condensed Matter 345, 89 (2004).

[38] I. A. Campbell, J. Phys. C: Solid State Phys. 1, 687 (1968).

[39] B. T. Thole, P. Carra, F. Sette, and G. van der Laan, Phys. Rev. Lett. 68, 1943 (1992).

[40] P. Carra, B. T. Thole, M. Altarelli, and X. Wang, Phys. Rev. Lett. 70, 694 (1993).

[41] O. Isnard and D. Fruchart, J. Alloys Compd. 205, 1 (1994).

[42] Y. Wang and J. P. Perdew, Phys. Rev. B 44, 13298 (1991).

[43] A. V. Ruban and H. L. Skriver, Comp. Mat. Science 15, 119 (1999).

[44] I. Turek, V. Drchal, J. Kudrnovský, M. Šob, and P. Weinberger, in Electronic Structure of Disordered Alloys, Surfaces and Interfaces (Kluwer Academic, Boston, 1997).

[45] A. I. Liechtenstein, M. I. Katsnelson, V. P. Antropov, and V. A. Gubanov, J. Magn. Magn. Mater. 67, 65 (1987).

[46] A. V. Ruban, S. I. Simak, S. Shallcross, and H. L. Skriver, Phys. Rev. B 67, 214302 (2003).

[47] A. V. Lukoyanov, E. E. Kokorina, M. V. Medvedev, and I. A. Nekrasov, Phys. Rev. B 80, 104409 (2009).

[48] E. A. Tereshina, D. I. Gorbunov, A. V. Andreev, and K. Watanabe, IEEE Trans. Magn. 47, 3610 (2011).

[49] O. Moze, R. Caciuffo, B. Gillon, G. Calestani, F. E. Kayzel, and J. J. M. Franse, Phys. Rev. B 50, 9293 (1994).

[50] I. Dzyaloshinskii, J. Phys. Chem. Solids 4, 241 (1958).

[51] T. Moriya, Phys. Rev. 120, 91 (1960).

[52] C. D. Hu, J. Phys.: Condens. Matter 24, 086001 (2012).

[53] P. Bak and M. H. Jensen, J. Phys. C: Solid St. Phys. 13, L881 (1980). 